\title{
Atresia de Coanas Associada ao Uso de Tapazol na Gestação
}

$\mathrm{R}$ ELATOS SUGEREM QUE ANOMALIAS congênitas graves podem ocorrer em fetos expostos ao metimazol (MTZ) durante a gestação (1). Relatamos o caso de um recém-nascido de mãe portadora de doença de Graves e diabetes mellitus tipo 1, tratada com $20 \mathrm{mg}$ de MTZ durante o primeiro trimestre, com exames realizados em torno da quarta semana, quando ainda se desconhecia a gestação, demonstrando bom controle glicêmico e da função tireoidiana (glico-hemoglobina: 6,0\%, frutosamina $1,78 \mathrm{mg} / \mathrm{dl}$ e TSH: 4,25 $\mu \mathrm{IU} / \mathrm{ml}$ ) até o final da gestação. Propiltiouracil (PTU) foi iniciado em substituição ao MTZ na 14a semana de gravidez. Criança nascida com 36 semanas de gestação com quadro clínico, laboratorial e radiológico compatível com doença da membrana hialina, que respondeu bem ao tratamento com surfactante. Após a não progressão da sonda nasal foi suspeitado de atresia de coanas, confirmada por tomografia de seios da face e fibronasolaringoscopia. O recémnascido apresentava ainda lesão hipocrômica em região parietal esquerda, compatível com aplasia cútis, não confirmada por biópsia, com regressão progressiva.

O PTU e o MTZ têm sido usados na gravidez e são igualmente efetivos no controle do hipertireoidismo (2). As teorias sugerindo que o PTU apresenta menor transferência placentária para o feto devido à maior ligação com a albumina plasmática não são sustentadas pela literatura atual (3). Permanece incerto se a doença de Graves não tratada ou não controlada aumenta o risco de malformações fetais (4). Diabetes mellitus tipo 1 não tem sido relacionado com atresia de coanas.

Há relatos de anomalias congênitas relacionada ao metimazol ( 8 casos) quando usado no início da gestação (época da organogênese fetal), que incluiria atresia de coanas, fístula esôfago-traqueal, dismorfismo facial, aplasia cútis e retardo do crescimento e do desenvolvimento psicomotor (5), não descritas com a exposição fetal ao PTU. A baixa prevalência dessas malformações na população leva ao questionamento do real risco do uso do $\mathrm{MTZ}$ na gestação e a sua relação com anomalias fetais.

Devido às infreqüentes, porém possíveis, associações de anomalias congênitas com o uso do MTZ, este se torna uma opção pouco atraente como terapia de primeira linha na doença de Graves durante a gestação, podendo ser considerada como droga de escolha no caso de intolerância, alergia ou falha no tratamento com PTU.

\section{REFERÊNCIAS}

1. Mandel S, Cooper D. The use of antithyroid drugs in pregnancy and lactation. J Clin Endocrinol Metab 2001;86:2354-9.

2. Wing DA, Millar LK, Koonings PP, Montoro MN, Mestman JH. A comparison of propyIthiouracil versus methimazole in the treatment of hyperthyroidism in pregnancy. Am J Obstet Gynecol 1997;170:90-5. carta ao editor

Tiago Alvarenga Fagundes

JaNICE SEPÚlVEDA REIS

PEDRo W. SOUza do Rosário

paulo A. Miranda Carvalho

SAULO PURISCH

Serviço de Endocrinologia e Metabologia da Santa Casa de Belo Horizonte, MG.
Recebido em 04/07/07

Aceito em 17/07/07 
Fagundes et al.

3. Mortimer RH, Cannell GR, Addison RS, Johnson LP, Roberts MS, Bernus I. Methimazole and propylthiouracil equally cross the perfused human term placental lobule. J Clin Endocrinol Metab 1997;82:3099-102.

4. Chattaway JM, Klepser TB. Propylthiouracil versus methimazole in treatment of Graves' disease during pregnancy. Ann Pharmacother 2007;41(6):1018-22.

5. Clementi M, Di Gianantonio E, Pelo E, Mammi I, Basile RT, Tenconi R. Methimazole embryopathy: delineation of the phenotype. Am J Med Genet 1999;83:43-6.

\section{Endereço para correspondência:}

Tiago Alvarenga Fagundes

Centro de Estudos e Pesquisa

Clínica de Endocrinologia e Metabologia (CEPCEM)

Av. Francisco Sales $1111,5^{\circ}$ andar, ala D

30150-221 Belo Horizonte, MG

Fax: (31) 3213-0836

E-mail: tifagundes@hotmail.com 